\title{
An Automated Approach to Differentiate Drug Resistant Tuberculosis in Chest X-ray Images Using Projection Profiling and Mediastinal Features
}

\author{
Sukanta Kumar TULO ${ }^{\mathrm{a}, 1}$, Palaniappan RAMU ${ }^{\mathrm{b}}$ and Ramakrishnan SWAMINATHAN ${ }^{\mathrm{a}}$ \\ ${ }^{\mathrm{a}}$ Department of Applied Mechanics, ${ }^{\mathrm{b}}$ Department of Engineering Design \\ Indian Institute of Technology Madras, Chennai, Tamil Nadu, India
}

\begin{abstract}
In this study, an attempt has been made to differentiate Drug Resistant Tuberculosis (DR-TB) in chest X-rays using projection profiling and mediastinal features. DR-TB is a condition which is non-responsive to at least one of anti-TB drugs. Mediastinum variations can be considered as significant image biomarkers for detection of DR-TB. Images are obtained from a public database and are contrast enhanced using coherence filtering. Projection profiling is used to obtain the feature lines from which the mediastinal and thoracic indices are computed. Classification of Drug Sensitive (DS-TB) and DR-TB is performed using three classifiers. Results show that the mediastinal features are found to be statistically significant. Support vector machine with quadratic kernel is able to provide better classification performance values of greater than $93 \%$. Hence, the automated analysis of mediastinum could be clinically significant in differentiation of DR-TB.
\end{abstract}

Keywords. Tuberculosis, drug resistant, mediastinum, chest X-ray

\section{Introduction}

Drug Resistant Tuberculosis (DR-TB) condition is the form of TB which is nonresponsive to at least one of anti-TB drugs [1]. Mediastinum is the central region of thorax surrounded by lungs laterally and hence considered to be a significant region for detection of DR-TB. Chest X-ray (CXR) is the preferred imaging modality for mass screening of the disease. Automated analysis of mediastinum in CXRs could aid in differentiation of DR-TB. In this study, an attempt has been made to differentiate Drug Sensitive (DS) and DR-TB in CXRs using projection profiling and mediastinal features.

\section{Methods}

For this study, 50 DS-TB and 50 DR-TB CXR images are considered from TB DEPOT, a public database [2]. The gray scale images with resolution of $256 \times 256$ pixels are contrast enhanced using coherence filtering for edge enhancement. Projection profiling

\footnotetext{
${ }^{1}$ Sukanta Kumar Tulo, Department of Applied Mechanics, Indian Institute of Technology Madras, Chennai, Tamil Nadu, India - 600036; E-mail: sukant.99t@gmail.com.
} 
is performed based on averaged horizontal projection to obtain the mediastinal feature lines. Mediastinal features namely, left mediastinal width, mediastinal width, thoracic width, Mediastinal Width Ratio (MWR) [3] and mediastinal thoracic ratio are extracted.

Linear Discriminant Analysis (LDA) and Support Vector Machine (SVM) [1] with different kernels have been reported for differentiation of TB conditions. Kernels in SVM transform a non-linear decision surface to a linear equation in higher dimensions. LDA, SVM with Linear kernel (SVM-Linear) and SVM with Quadratic kernel (SVMQuadratic) are employed for differentiation of DS-TB and DR-TB. The class specific performance of classifiers is evaluated using sensitivity, precision and F-measure [1].

\section{Results and Discussion}

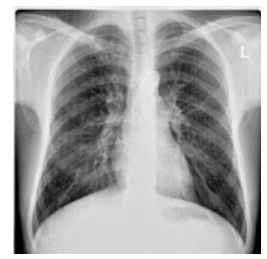

(a)

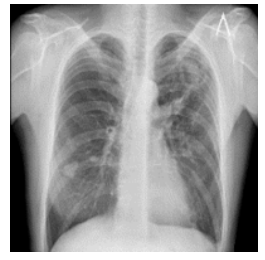

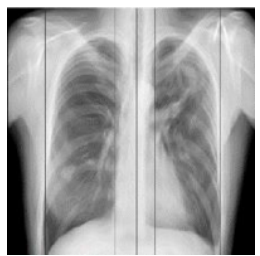

(b)

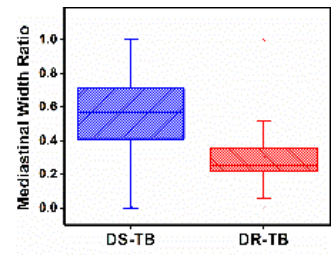

(c)

Figure 1. Representative (a) DS-TB and DR-TB CXRs; (b) Feature lines on DR-TB; and (c) MWR plot.

From Figure 1, it is observed that raw CXRs suffer from poor edge contrast. Projection profiling method is able to extract the mediastinal features from the coherence filtered CXR image. Extracted features are found to be statistically significant $(p<0.05)$. A significant distinction is also observed between DS-TB and DR-TB from the MWR plot. From Table 1, it is seen that the maximum F-measure of $93.6 \%$ for both the classes is obtained using SVM-Quadratic. LDA is able to provide maximum sensitivity of $96.0 \%$ for DR-TB while SVM-Quadratic provides maximum sensitivity of $93.3 \%$ for DS-TB. From the results, the performance of the proposed automated approach is found to be higher than the existing methods to differentiate DR-TB [1]. Hence, this study will be useful to assist the clinicians for early detection of DR-TB.

Table 1. Class specific comparison of classification performance

\begin{tabular}{ccccccc}
\hline \multirow{2}{*}{ Classifiers } & \multicolumn{2}{c}{ Sensitivity (\%) } & \multicolumn{2}{c}{ Precision (\%) } & \multicolumn{2}{c}{ F-measure (\%) } \\
& DS-TB & DR-TB & DS-TB & DR-TB & DS-TB & DR-TB \\
\hline LDA & 80.0 & $\mathbf{9 6 . 0}$ & $\mathbf{9 5 . 0}$ & 82.7 & 86.8 & 88.8 \\
SVM-Linear & 84.7 & 93.3 & 92.3 & 85.7 & 88.3 & 89.3 \\
SVM-Quadratic & $\mathbf{9 3 . 3}$ & 94.0 & 94.0 & $\mathbf{9 3 . 3}$ & $\mathbf{9 3 . 6}$ & $\mathbf{9 3 . 6}$ \\
\hline
\end{tabular}

\section{References}

[1] Jaeger S, Juarez-Espinosa OH, Candemir S, Poostchi M, Yang F, Kim L, Ding M, Folio LR, Antani S, Gabrielian A, Hurt D. Detecting drug-resistant tuberculosis in chest radiographs. Int J Comput Assist Radiol Surg. 2018 Dec;13(12):1915-25.

[2] Gabrielian A, Engle E, Harris M, Wollenberg K, Juarez-Espinosa O, Glogowski A, Long A, Patti L, Hurt DE, Rosenthal A, Tartakovsky M. TB DEPOT (Data Exploration Portal): A multi-domain tuberculosis data analysis resource. Plos One. 2019 May;14(5):e0217410.

[3] Lai V, Tsang WK, Chan WC, Yeung TW. Diagnostic accuracy of mediastinal width measurement on posteroanterior and anteroposterior chest radiographs in the depiction of acute nontraumatic thoracic aortic dissection. Emerg Radiol. 2012 Aug;19(4):309-15. 